\title{
Formalizing Interoperability Testing: Quiescence Management and Test Generation
}

\author{
Alexandra Desmoulin and César Viho \\ IRISA/Université de Rennes 1, Campus de Beaulieu, \\ 35042 Rennes Cedex, France \\ \{adesmoul, viho\}@irisa.fr \\ http://www.irisa.fr/armor
}

\begin{abstract}
This paper gives formal definitions of the different existing interoperability notions called interoperability criteria. The equivalence between two of them leads to a method for interoperability test generation that avoids the state explosion problem of classical approaches.
\end{abstract}

\section{Introduction}

Despite a large literature on the interest of providing a formal approach for interoperability testing [1,2, only few tentative have been proposed. Therefore, the aims of this study presented in this paper are double. First, we give formal definitions of interoperability testing called interoperability criteria (iop criteria for short in the following). The second contribution of this work is a new method to generate automatically interoperability test cases. It uses a theorem proving the equivalence between two iop criteria. It avoids the well-known state-explosion problem due to the classical construction of the specification composition. Thus, the proposed method is a real solution that provides an easy and efficient way to derive effectively interoperability test cases.

\section{Interoperability Definitions}

One-to-One Interoperability Testing Architecture. In this study, we consider the one-to-one interoperability context : the System Under Test (SUT) is composed of two Implementation Under Test (IUT). There are two kind of interfaces. The lower interfaces used for the interaction of the IUTs and the upper interfaces used for the communication with their environment. Depending on the access to the different interfaces, different architectures can be distinguished.

Formal Background. The well-known IOLTS (Input-Output Labeled Transition System) model will be used to model specifications and to define interoperability criteria. We note $p ? m(p ! a)$ for an input (output) of message $m$ on the interface $p$. Figure 1 gives an example of two specifications using this model.

Quiescence and ioco. Three main situations lead to quiescence of a system : deadlock (a state after which no event is possible), outputlock (a state after which 


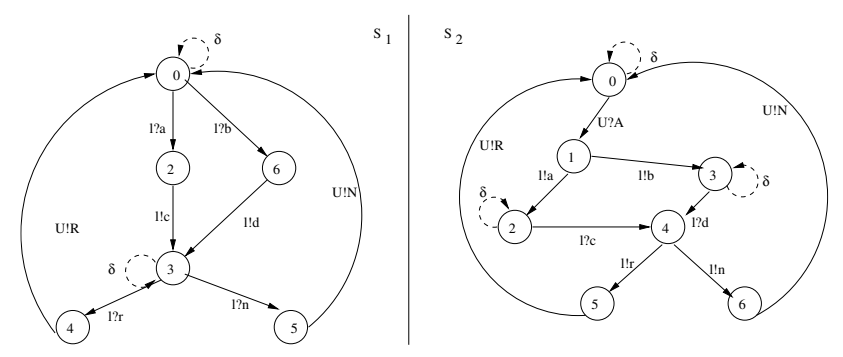

Fig. 1. Specifications $S_{1}$ and $S_{2}$

only transitions labeled with input exist) and livelock (a loop of internal events). Quiescence is modeled by $\delta$ and is treated as an observable output event. The obtained IOLTS is called suspensive IOLTS [3] and noted $\Delta(M)$. The ioco conformance relation [3] is used for the formal interoperability definitions. It says that an IUT $I$ is ioco-conformant to its specification $S$ if $I$ can never produce an output that could not be produced by $S$ after the same suspension trace.

Interaction. We need a model of the asynchronous interaction of the implementations. This is noted $M_{1} \|_{\mathcal{A}} M_{2}$ and obtained as usual by a synchronous composition of $\Delta\left(M_{1}\right), \Delta\left(M_{2}\right)$ and FIFO queues modeling the asynchronous environment. Quiescence is preserved and $\delta(i)$ corresponds to quiescence of $M_{i}$ and $\delta$ of the two IOLTS.

Projection. In interoperability testing, we usually need to observe some specific events of an IUT. $M / X$ represents the projection of the behavior of the implementation $M$ reduced to a set $X$ of expected messages.

Model of an Implementation: iop-Input Completion. In the context of interoperability testing, tester can only observe the events on the lower interfaces. But these testers can not differenciate events received by an IUT from events effectively treated. A completion is needed for inputs corresponding to the output alphabet of the other IUT specification. It is called the iop-input completion leading the IOLTS into an error deadlock state.

Formal Definition of Interoperability Criteria. According to the chosen testing architecture, different notions of interoperability can be used [4]. We will focus here on two interoperability (iop) criteria. The global iop criterion iop $_{G}$ says that two implementations are considered interoperable if, after a suspensive trace of the asynchronous interaction of the specifications, all outputs and quiescence observed during the asynchronous interaction of the implementations are foreseen in the specifications. The bilateral iop criterion $i_{0} p_{B}$ says that after a suspensive trace of $S_{1}$ observed during the asynchronous interaction of the implementations, all outputs and quiescence observed in $I_{1}$ are foreseen in $S_{1}$, and the same in the point of view of $I_{2}$ implementing the specification $S_{2}$.

The most important result is the following theorem 1] stating that $\operatorname{iop}_{G}$ is equivalent to the bilateral total iop criterion $i o p_{B}$.

Theorem 1. $I_{1} \operatorname{iop}_{G} I_{2} \Leftrightarrow I_{1} \operatorname{iop}_{B} I_{2}$ 


\section{Interoperability Test Generation}

The goal of an interoperability test generation algorithm is to generate interoperability Test Cases $(T C)$ that can be executable on the SUT composed of the two IUT to be tested. The inputs of such algorithms are the specifications $S_{1}$ and $S_{2}$ on which the two IUT $\left(I_{1}\right.$ and $\left.I_{2}\right)$ are based, and a Test Purpose $(T P)$ which is a particular property (in the shape of incomplete sequences of actions that have to be observed or sent to the SUT) to be tested.

Interoperability Verdicts. The execution of an iop test case $T C$ on $S U T\left(I_{1} \|_{\mathcal{A}} I_{2}\right)$ gives a verdict : verdict $(T C, S U T) \in\{P A S S, F A I L, I N C\}$. The interoperability verdict PASS means that no interoperability error was detected, FAIL means that the iop criterion is not verified, and INC (for Inconclusive) means that the behavior of the SUT seems valid but it is not the purpose of the test case.

The Classical Approach and the State-Space Explosion Problem. In the classical approach based on a criteria like $i o p_{G}$, the test generation algorithm begins with the construction of the asynchronous interaction $S_{1} \|_{\mathcal{A}} S_{2}$. Then $S_{1}$ $\|_{\mathcal{A}} S_{2}$ is composed with the TP. The consistency of $T P$ is checked in parallel and $T C$ is generated. Yet, the construction of $S_{1} \|_{\mathcal{A}} S_{2}$ can cause the well-known state-space explosion, as building $S_{1} \|_{\mathcal{A}} S_{2}$ is exponential in the number of states of $S_{1}$ and $S_{2}$ and the FIFO queues size. Thus, interoperability test generation based on the global iop criterion may be impossible even for small specifications.

A New Method Based on the Bilateral iop Criterion $i_{B} p_{B}$. The equivalence of $i o p_{B}$ and $i o p_{G}$ (cf. therorem 11) suggests to study a method for iop test cases generation based on the bilateral iop criterion $i o p_{B}$. The idea is to derive $T P_{S_{i}}$ from an iop test purpose $T P$. Each $T P_{S_{i}}$ represents $T P$ in the point of view of $S_{i}$. This step is described in the following algorithm (see figure 2). The second step is to use a conformance test generation tool $\mathcal{F}$ such that $\mathcal{F}:\left(S_{1}\right.$, $\left.T P_{S_{1}}\right) \rightarrow T C_{1}$ and $\mathcal{F}:\left(S_{2}, T P_{S_{2}}\right) \rightarrow T C_{2}$. We obtain two unilateral iop test cases $T C_{1}$ and $T C_{2}$. The obtained test cases obtained are modified in order to take into account the differences between upper and lower interfaces in interoperability testing. For example, an event $l ! m$ (resp. $l ? m$ ) in the obtained test case will be replaced by ?(l?m) (resp. ? $(l ! m)$ ) in the interoperability test case. This means that the unilateral interoperability tester observes that a message $m$ is received from (resp. sent to) the other IUT on the lower interface $l$. No changes are made on the test cases for events on the upper interfaces. According to the theorem 1, verdict $\left(T C, I_{1} \|_{\mathcal{A}} I_{2}\right)=\operatorname{verdict}\left(T C_{1}, I_{1} \|_{\mathcal{A}} I_{2}\right) \wedge \operatorname{verdict}\left(T C_{2}, I_{1}\right.$ $\left.\|_{\mathcal{A}} I_{2}\right)$. The rules for the combination of these two verdicts to obtain the final iop $_{B}$ verdict are given by : $P A S S \wedge P A S S=P A S S, P A S S \wedge I N C=I N C$, $I N C \wedge I N C=I N C$, and $F A I L \wedge(F A I L \vee I N C \vee P A S S)=F A I L$.

Applying the Method to an Example. Let us consider the two specifications $S_{1}$ and $S_{2}$ of figure 1 and the interoperability testing purpose $T P=l 1 ? a . U 2 ! N$. This test purpose is interesting because it contains events on both interfaces 
Input: $T P$ : test purpose; Output: $\left\{T P_{S_{i}}\right\}_{i=1,2}$;

Invariant: $S_{k}=S_{3-i}\left({ }^{*} S_{k}\right.$ is the other specification $\left.{ }^{*}\right) ; T P=\mu_{1} \ldots \mu_{n}$

Initialization: $\mu_{0}=\epsilon ; T P_{S_{i}}=\epsilon$;

for $(\mathrm{i}=0 ; i \leq n ; \mathrm{i}++)$ do

if $\left(\mu_{i} \in \Sigma^{S_{i}}\right)$ then $T P_{S_{i}}=T P_{S_{i}} \cdot \mu_{i}$ (* just add $\left.{ }^{*}\right)$

if $\left(\mu_{i} \in \Sigma_{L}^{S_{k}}\right)$ then $T P_{S_{i}}=T P_{S_{i}} \cdot \overline{\mu_{i}}$ (* just add the mirror $\left.{ }^{*}\right)$

if $\left(\mu_{i} \in \Sigma_{U}^{S_{k}} \cup\{\tau\}\right)$

$\sigma_{1}:=T P_{S_{i}} ; a_{j}=$ last_event $\left(\sigma_{1}\right)$

while $a_{j} \in \Sigma_{U}^{S_{k}} \cup\{\tau\}$ do $\sigma_{1}=$ remove_last_event $\left(\sigma_{1}\right)$

$a_{j-1}=$ last_event $\left(\sigma_{1}\right)\left(* a_{j-1}\right.$ is the last event added to $T P_{S_{i}}$ and

end a mirror event $\bar{a}_{j-1}$ may exist in $S_{k} *$ )

$M_{S_{k}}=\left\{q \in Q^{S_{k}}\right.$ such that $\mathrm{q} \stackrel{\bar{a}_{j-1}}{\rightarrow}$ and $\left.\sigma=\bar{a}_{j-1} \cdot \omega \cdot \mu_{i} \in \operatorname{Traces}(q)\right\}$

if $\left(\forall q \in M_{S_{k}}, q \stackrel{\sigma}{\longrightarrow}\right)$ then error(TP not valid : no path to $\left.\mu_{i}\right)$

while (e=last_event $\left.(\omega) \notin \Sigma_{L}^{S_{k}} \cup\{\epsilon\}\right)$ do $\omega=$ remove_last_event $(\omega)$ end

if $\left(\mathrm{e} \in \Sigma_{L}^{S_{k}}\right)$ then $T P_{S_{i}}=T P_{S_{i}} \cdot \bar{e}$

else error(TP not valid : $\mu_{i} \notin \Sigma^{S_{1}} \cup \Sigma^{S_{2}}$ )

Fig. 2. Algorithm to derive $T P_{S_{i}}$ from $T P$

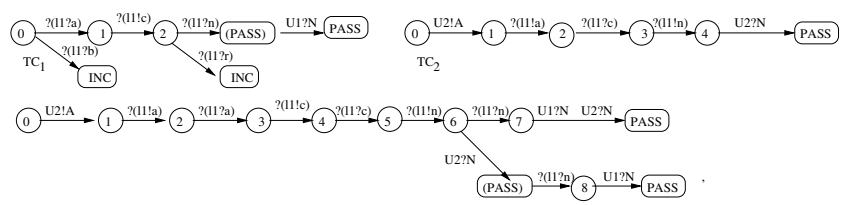

Fig. 3. Interoperability test cases obtained for $T P=l 1 ? a \cdot U 2 ! N$

and both IUTs. Applying the algorithm of figure 2, we obtain : $T P_{S_{1}}=l 1$ !a.l1?n and $T P_{S_{2}}=\bar{\mu}_{1} \cdot \mu_{2}=l 2 ! a . U 2 ! N$. The obtained test cases $T C_{1}$ and $T C_{2}$ are given in upper side of figure 3. For interoperability test case generation based on the global relation, the obtained $T C$ (cf. the third test case in figure 3) comes from the composition of $S_{1} \|_{\mathcal{A}} S_{2}$ with $T P$. According to the theorem 11, final interoperability verdicts obtained with $T C_{1}$ and $T C_{2}$ should be the same as the verdict obtained with $T C$. The proof is not given here but a look at glance to $T C_{1}$ and $T C_{2}$ shows the same paths and verdicts in $T C$.

\section{Conclusion}

In this paper, interoperability criteria taking quiescence into account are defined, describing the conditions under which two IUT can be considered interoperable. A theorem proving that two of them are equivalent allows a new method for interoperability test generation that avoids the classical state-explosion problem. Further studies will consider a distributed approach for interoperability testing of architectures composed of more than two implementations. 


\section{References}

[1] O. Rafiq and R. Castanet. From conformance testing to interoperability testing. In Protocol Test Systems, volume III, pages 371-385, North-Holland, 1991. IFIP, Elsevier sciences publishers B. V.

[2] S. Seol, M. Kim, S. Kang, and J. Ryu. Fully automated interoperability test suite derivation for communication protocols. Comp. Networks, 43(6):735-759, 2003.

[3] J. Tretmans. Testing concurrent systems: A formal approach. In J.C.M Baeten and S. Mauw, editors, CONCUR'99 $-10^{\text {th }}$ Int. Conference on Concurrency Theory, volume 1664 of LNCS, pages 46-65. Springer-Verlag, 1999.

[4] S. Barbin, L. Tanguy, and C. Viho. Towards a formal framework for interoperability testing. In M. Kim, B. Chin, S. Kang, and D. Lee, editors, FORTE' 2001, pages 53-68, Cheju Island, Korea, 2001. 